\title{
Educational Technology Research with International Perspectives and Cultural Contexts
}

\author{
Yu-Chang Hsu ${ }^{1}$ - Charles Xiaoxue Wang ${ }^{2}$
}

Published online: 2 May 2019

(C) Association for Educational Communications \& Technology 2019

This special issue of TechTrends, sponsored by the International Division of Association for Educational Communications and Technology (AECT), aims to provide a rich snapshot of the current landscape of educational technology research through the lenses of international perspectives and cultural contexts. As a professional organization, AECT has a diverse membership from around the globe with a long history of promoting and facilitating collaborative research efforts among its members in a worldwide arena. Part of AECT's unique characteristic is the outlet of a collaborative framework for educational technology innovation and diffusion for learning and research shared in every part of the world.

Global perspectives are of great value to inform and inspire educational technology research that recognizes and respects rich cultural differences and unique local contexts. This special issue sought articles that (1) examine international perspectives on research in educational technology, (2) report collaborative research efforts across geographic distance between and among different nations, (3) discuss technology and learning related theoretical concepts that promote and facilitate international collaborations, (4) explore professional ethics, unique cultures, and policies related to educational technology in a global arena, and (5) share innovative ways of meeting educational technology related challenges in the authors' own countries. This special issue includes a variety of topics, in and across diverse geographical regions highlighting the themes mentioned above.

Yu-Chang Hsu

hsu@boisestate.edu

Charles Xiaoxue Wang

xxwang@fgcu.edu

1 Boise State University, Boise, ID, USA

2 Florida Gulf Coast University, Fort Myers, FL, USA
In the first article, Xie, Ryder, and Chen explored college students' use of interactive virtual reality (VR) tools for learning Chinese as a foreign language. The study indicated that the real-life view VR tools offered an authentic context for Chinese language learning, sparked interest in the virtually presented locales, encouraged students to further explore the target culture, and enhanced student engagement in language learning. This study illuminated one possible way of how VR tools can be used to facilitate learning of a foreign language.

Hsu, Irie, and Ching studied the representative computational thinking (CT) educational policy initiatives through the lens of international perspectives and cultural contexts. They found that Computational Thinking Educational Policy Initiatives (CTEPI) has two major characteristics: Diverse CT terminology and diverse status across the globe, and revealed curriculum reform and enrichment programs as two major approaches for CTEPI. Their article identified four development trends of $\mathrm{CT}$ education policies and initiatives: Collaboration and partnerships across sectors and national boundaries, rationales taking a broad perspective and referring to common themes, a redefinition of digital competence, and an emphasis on broadening access and interest.

Park, Ryu, and McChesney described a collaborative simulation design studio between two universities in South Korea and the U.S. They described the design of the studio program and activities, in which the pre-service teachers from two countries created four classroom management scenarios for virtual students/classrooms. The design experiences helped participants understand different classroom cultures according to their responses to the reflection questions, evaluation of design outcomes, and the researchers' observation notes. The two major themes emerging from participants' responses to cultural differences and similarities are classroom management and student attitude. Social engagement and tangible experiences were the perceived benefits of the collaborative design studio experiences.

Mao, Ifenthaler, Fujimoto, Garavaglia, and Rossi collaborated to explore the trends and perspectives on educational 
technology in China, Germany, Italy, Japan, and the United States, by using the STEEP (social, technological, economic, environmental and political) framework. They systematically studied the relevant policies of these nations and discussed their influence of on educational technology implementation and research. They also explored the local constraints on the adoption of and research on innovation to highlight the strategies and reforms that different countries have adopted to prepare the next generation for the constantly changing, globalized twenty-first century.

Breman, Giacumo, and Griffith-Boyes described a needs analysis case study to inform the instructional design of a multinational capacity building project in humanitarian logistics. Survey responses from 106 foreign partner nongovernmental organizations (NGOs) were collected to gain insights into organizations' access to technology, levels of confidence in five logistics process areas, the strengths of previous capacity building projects, and organizational strategies and methods for capacity building. Results showed that staff have access to both mobile phones and computers and preferred to use the latter for learning. Also, respondents preferred participatory approaches to scenario-based training over lecture-style presentations.

Grossman shared a video production project that combined applied cognitive science and participatory video methodology to design and create high-quality skill-learning videos for adults in The Gambia, West Africa. She reflected upon the collaboration of the project design and development and reviewed the characteristics of the videos created. The project merits were examined through interviews with villagers, which revealed that village women valued video education because learning was easily accessible and the skills presented had benefits for their lives.

Rich, Browning, Perkins, Shoop, Yoshikawa, and Belikov provided a broad picture of teaching computing in primary education from teachers' perspectives through a 20-min snowball survey sent out to elementary computing groups. They reported on the teaching of over 300 teachers who were responsible for teaching computing to nearly 60,000 primaryaged students in 23 countries. The results highlighted teachers' level of preparation to teach computing, the most commonly taught programming languages, teacher successes and challenges, and observations on how learning to program has affected students.
Torres-Barzabal, del Pilar Ortiz-Calderón, and BarciaTirado discussed a model for the assessment of the quality of online teaching based on an audit by specialized technical personnel. The personnel were independent of the design of the teaching materials which could be a proposal for the higher education quality assurance models of the European Association for Quality Assurance in Higher Education (ENQA). The authors proposed that the template could be useful in undergraduate and graduate courses. The instrument developed for the audits includes all the criteria and indicators considered relevant for quality assessment from a pedagogical perspective.

Aldosemani, Shepherd, and Bolliger discussed Saudi faculty members' perceptions of blended learning at an emerging university in central Saudi Arabia. They documented the support and challenges that instructors encounter as they implement blended approaches. Suggestions regarding faculty training and reward structures were provided to promote blended learning in Saudi Arabia.

We are very grateful for the full support received from the International Division board members as well as general members. As guest editors, we especially appreciate Dr. Charles Hodges, Editor-in-Chief of TechTrends, for his leadership, professional guidance, and shared vision as we worked on this special issue. We are able to celebrate this milestone because of his timely and instrumental support. In addition, we want to express our sincere gratitude to Dr. Michele Garabedian Stork, Dr. Vickie Johnston, and Joann Ellis, a doctoral student, at Florida Gulf Coast University for spending their weekends and winter break to help us with editing and proofreading. Their dedicated work with professional expertise and well-thought-out suggestions to us contributed tremendously to the quality of this special issue. Finally, we are truly honored and blessed to work as guest editors of this special issue and to engage in this ever-going momentum of the intellectual exchange with every author. With your contributions, we celebrate together the publication of this special issue for educational technology research with international perspectives and cultural contexts across the globe.

Publisher's Note Springer Nature remains neutral with regard to jurisdictional claims in published maps and institutional affiliations. 